OPEN ACCESS

Edited by:

Diego Gallo,

Politecnico di Torino, Italy

Reviewed by:

Kyeong Ho Yun,

Wonkwang University, South Korea

Felice Gragnano,

University of Campania Luigi

Vanvitelli, Italy

*Correspondence:

Linping $L i$

IIp0522@163.com

Specialty section:

This article was submitted to Cardiovascular Therapeutics,

a section of the journal

Frontiers in Cardiovascular Medicine

Received: 19 November 2021

Accepted: 24 December 2021

Published: 27 January 2022

Citation:

Sun M, Cui W and Li L (2022)

Comparison of Clinical Outcomes Between Ticagrelor and Clopidogrel in

Acute Coronary Syndrome: A

Comprehensive Meta-Analysis.

Front. Cardiovasc. Med. 8:818215.

doi: 10.3389/fcvm.2021.818215

\section{Comparison of Clinical Outcomes Between Ticagrelor and Clopidogrel in Acute Coronary Syndrome: A Comprehensive Meta-Analysis}

\author{
Mengyi Sun ${ }^{1}$, Weichen Cui ${ }^{2}$ and Linping $\mathrm{Li}^{3 *}$ \\ ${ }^{1}$ Department of Clinical Laboratory, Jining Academy of Medical Sciences, Jining, China, ${ }^{2}$ Department of Clinical Laboratory, \\ Jiaxiang Women and Children's Hospital, Jining, China, ${ }^{3}$ Department of Cardiology, Jining Academy of Medical Sciences, \\ Jining, China
}

Background: Ticagrelor is currently recommended for patients with the acute coronary syndrome (ACS). However, recent studies have yielded controversial results.

Objective: To compare the clinical outcomes between ticagrelor and clopidogrel in patients with ACS.

Methods: Three electronic databases were queried until April 25, 2021. We defined major adverse cardiovascular events (MACEs) as the primary efficacy endpoint. The secondary efficacy endpoints included stroke, stent thrombosis, cardiovascular death, all-cause death, and myocardial infarction. The safety endpoints were (major and minor) bleeding. Odds ratios (ORs) and 95\% Cls were calculated to represent the estimated effect sizes.

Results: A total of 270,937 patients with ACS from 10 clinical trials and 18 observational studies were included. No significant difference was detected in MACE (OR 0.81, 95\% $\left.\mathrm{Cl} 0.60-1.08, p=0.15, I^{2}=64.83 \%\right)$. However, ticagrelor introduced a higher risk of bleeding $(1.46,1.17-1.83,0.00,61.66 \%)$ and minor bleeding $(1.71,1.33-2.21,0.00$, $4.65 \%)$ in clinical trials. The results of secondary efficacy endpoints differed in the clinical trials and observational studies. Subgroup analysis demonstrated that ticagrelor showed better therapeutic effects in patients who underwent the percutaneous coronary intervention $(\mathrm{PCI})(0.38,0.23-0.63,0.00,0)$ than those intended for $\mathrm{PCl}(1.03,0.76-1.38$, $0.87,64.26 \%)$. Meanwhile, ticagrelor showed different therapeutic effects on patients with ACS of different ethnicities and different countries.

Conclusion: This meta-analysis demonstrated that ticagrelor is not superior to clopidogrel in MACE but is associated with a higher risk of bleeding in patients with ACS. Different PCl strategies, ethnicities, and countries may be the factors that contribute to different therapeutic effects of ticagrelor.

Systematic Review Registration: This study is registered with PROSPERO (CRD42021251212).

Keywords: ticagrelor, clopidogrel, acute coronary syndrome, percutaneous coronary intervention, meta-analysis 


\section{INTRODUCTION}

Currently, cardiovascular disease (CVD) is the largest contributor to the disease burden, accounting for approximately one-third of the global deaths (1). Besides, acute coronary syndrome (ACS), as a common and serious CVD, has a dramatically increased incidence with age, proposing a great challenge to public medical care. Dual antiplatelet therapy (DAPT) is the mainstay treatment strategy for ACS, with timely vascularization as needed $(2,3)$. As for the choice of antiplatelet agent, ticagrelor is recommended over clopidogrel for patients with ACS or who have received the percutaneous coronary intervention (PCI) in the 2016 American College of Cardiology (ACC)/American Heart Association (AHA) guidelines and the 2018 European Society of Cardiology (ESC)/European Association for Cardio-Thoracic Surgery (EACTS) guidelines $(2,4)$.

As a novel adenosine diphosphate receptor antagonist, ticagrelor provides faster, more potent, and more stable platelet inhibition than clopidogrel $(5,6)$. The large Platelet Inhibition and Patient Outcomes (PLATO) trial exhibited that compared with clopidogrel, ticagrelor reduced the incidences of stroke, myocardial infarction (MI), and cardiovascular (CV) death, without elevating the risk of major bleeding (7). However, other large clinical trials $(8,9)$ and observational studies $(10-$ 12) drew controversial conclusions. Meanwhile, several metaanalyses published recently also reported inconsistent results (13-16). Therefore, we conducted a meta-analysis to review previous relevant studies and compared the clinical benefits of ticagrelor and clopidogrel in the ACS population using aspirin to address these conflicting conclusions.

\section{METHODS}

This meta-analysis was conducted following the Preferred Reporting Items for Systematic Reviews and Meta-Analyses (PRISMA) guideline (17) (Supplementary Table S1) and has been registered in an international prospective register of systematic reviews PROSPERO (ID: CRD42021251212).

\section{Literature Search}

Three electronic databases, Cochrane, EMBASE, and PubMed library, were searched for eligible citations before April 25, 2021. The following keywords were applied: "ticagrelor," "clopidogrel," "myocardial ischemia," "ACS," "percutaneous coronary intervention," and "PCI." The detailed search strategy is shown in Supplementary Table S2. In addition, references in relevant meta-analyses were manually searched for potential eligibility.

\section{Inclusion and Exclusion Criteria}

We reviewed the full texts of these potentially eligible kinds of literature to determine whether they fulfilled the following inclusion criteria: (1) adult ( $\geq 18$ years old) patients with ACS who underwent PCI (PCI strategy proportion equal to 100\%) or intended for PCI (proportion less than 100\%), which were described in titles and abstracts or baseline characteristics forms;
(2) clinical trials and observational studies comparing ticagrelor vs. clopidogrel in the context of aspirin use; (3) one or more of the following outcomes reported during any follow-up period: MACE, all-cause death, CV death, MI, stroke, stent thrombosis (ST), and (major or minor) bleeding. Exclusion criteria were as follows: (1) studies with incomplete data, or observational studies with unadjusted endpoints; (2) studies from the same sample source; (3) studies not in English.

\section{Study Endpoints}

The primary efficacy endpoints were trial-defined primary MACEs or efficacy endpoints (described as death/CV death, MI, and/or stoke) (Supplementary Table S4). The secondary endpoints included stroke, ST, MI, CV death, and all-cause death. The safety endpoints were trial-defined bleeding (described as bleeding, major or minor bleeding) (Supplementary Table S4). For the definitions of safety outcomes, if not otherwise specified, we prioritized PLATO definitions when available (Supplementary Table S5).

\section{Data Extraction}

Data from the included citations were independently populated with a standardized data extraction by two researchers, with any discrepancy resolved by a third researcher. The data included the last names of authors, study type, country, publication year,

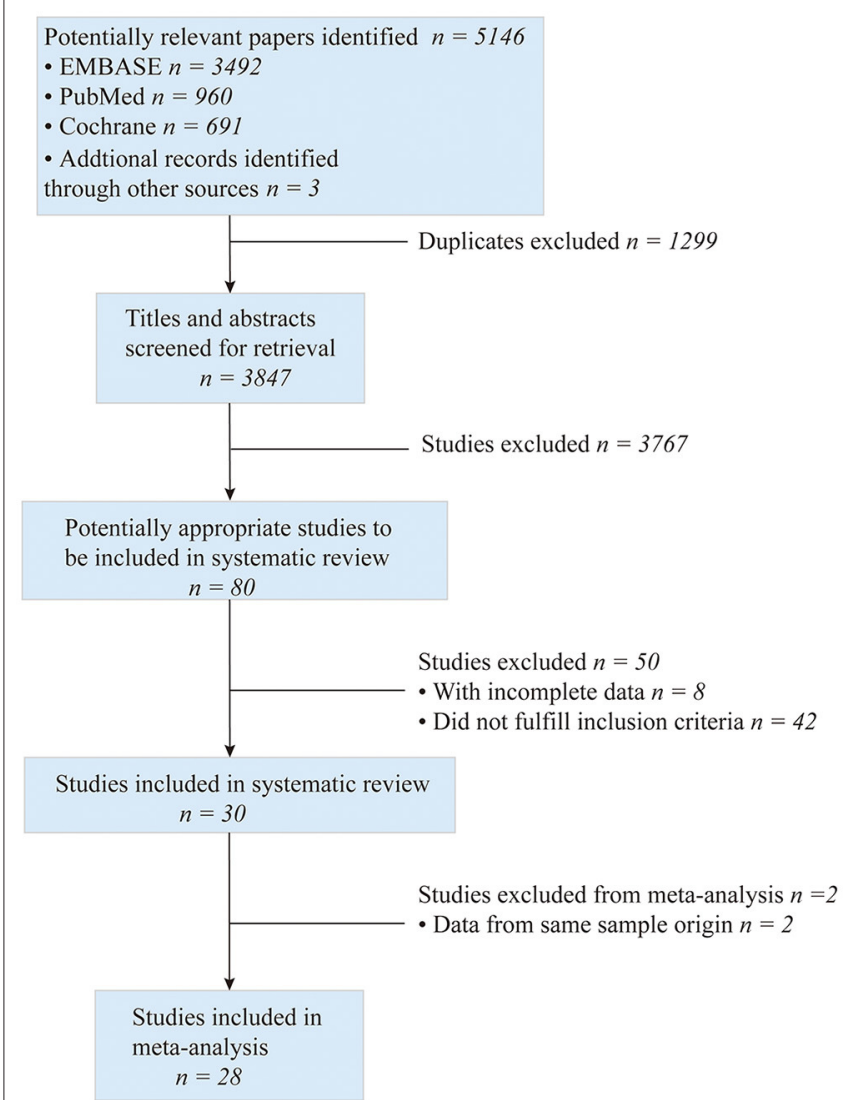

FIGURE 1 | Flowchart diagram of searching and screening of studies. 
journal, disease subtype, sample size, age, gender distribution, the PCI strategy, dosing regimen, reported outcomes about efficacy and safety, and follow-up duration.

\section{Quality Assessment}

We assessed the bias risk of randomized controlled trials (RCTs) using the Cochrane Collaboration risk-of-bias-tool (RoB 2) (18), which covers five domains of bias to classify the RCTs into three levels (low risk of bias, some concerns, and high risk of bias) (Supplementary Table S6). Meanwhile, we assessed the quality of observational studies using the Newcastle-Ottawa Scale (NOS), which includes eight items for three aspects (selection, comparability, and outcome) assessments (Supplementary Table S7). The NOS adopts the semiquantitative principle of star allocation to assess the literature quality, with a full score of nine stars (19).

\section{Statistical Analysis}

We used the odds ratios (ORs) and 95\% CIs to represent the estimated effect sizes, which were obtained via the Stata 16.0 software (StataCorp, CollegeStation, TX, USA) (20). Given the inclusion of heterogeneous populations, we chose the randomeffects model to pool the effect sizes for this meta-analysis. Furthermore, we used the Higgins' $I^{2}$ statistics and Cochran's $Q-$ test to estimate heterogeneity across studies. A $p$-value $<0.05$ was considered statistically significant.

All the analyses were performed by analyzing data from clinical trials and observational studies separately to reduce heterogeneity caused by different study types. Meanwhile, subgroup analyses were performed to search for potential sources of heterogeneity. In brief, according to different data types of the observational studies, we performed two subgroup analyses, namely, propensity score-matched/adjusted analyses (PA group) and multivariate-adjusted analyses (MA group). In addition, we conducted prespecified subgroup analyses on the included RCTs based on PCI strategy, ethnicity, country, duration of follow-up, and enrollment time.

The stability of our findings was evaluated by sensitivity analyses, which means to calculate the effects by including highquality RCTs and by only including studies with clinical event committee-adjudicated events in RCTs (21). When more than ten studies were included, the presence of publication bias was investigated by Egger's test and displayed by visual estimation (symmetry) of funnel plots.

\section{RESULTS}

\section{Eligible Studies and Patient Characteristics}

The process of searching, retrieving, and screening for this metaanalysis is shown in Figure 1. A total of 5,146 potentially relevant kinds of literature were screened. Then 1,299 duplicates were excluded, and 3,767 were retrieved for the title and abstract screening. Subsequently, the full texts of 80 articles were reviewed for eligibility. Among them, 42 articles did not fulfill the inclusion criteria, 8 presented incomplete data, and 2 employed the same cohorts included in our study. Finally, 28 studies with 270,937 patients $(88,490$ and 182,447 in the ticagrelor and clopidogrel groups, respectively) were included for the meta-analysis. There were 10 clinical trials $(5,7-9,22-27)$ and 18 observational studies (10-12, 28-42). The patients were enrolled from 2003 to 2019, and the articles were published from 2007 to 2020. Among them, 19 studies included patients with ACS who underwent PCI, while 9 included those intended for PCI. A total of 254,450 patients received PCI. The countries, in which these studies were conducted, included East Asian countries, such as China, Korea,

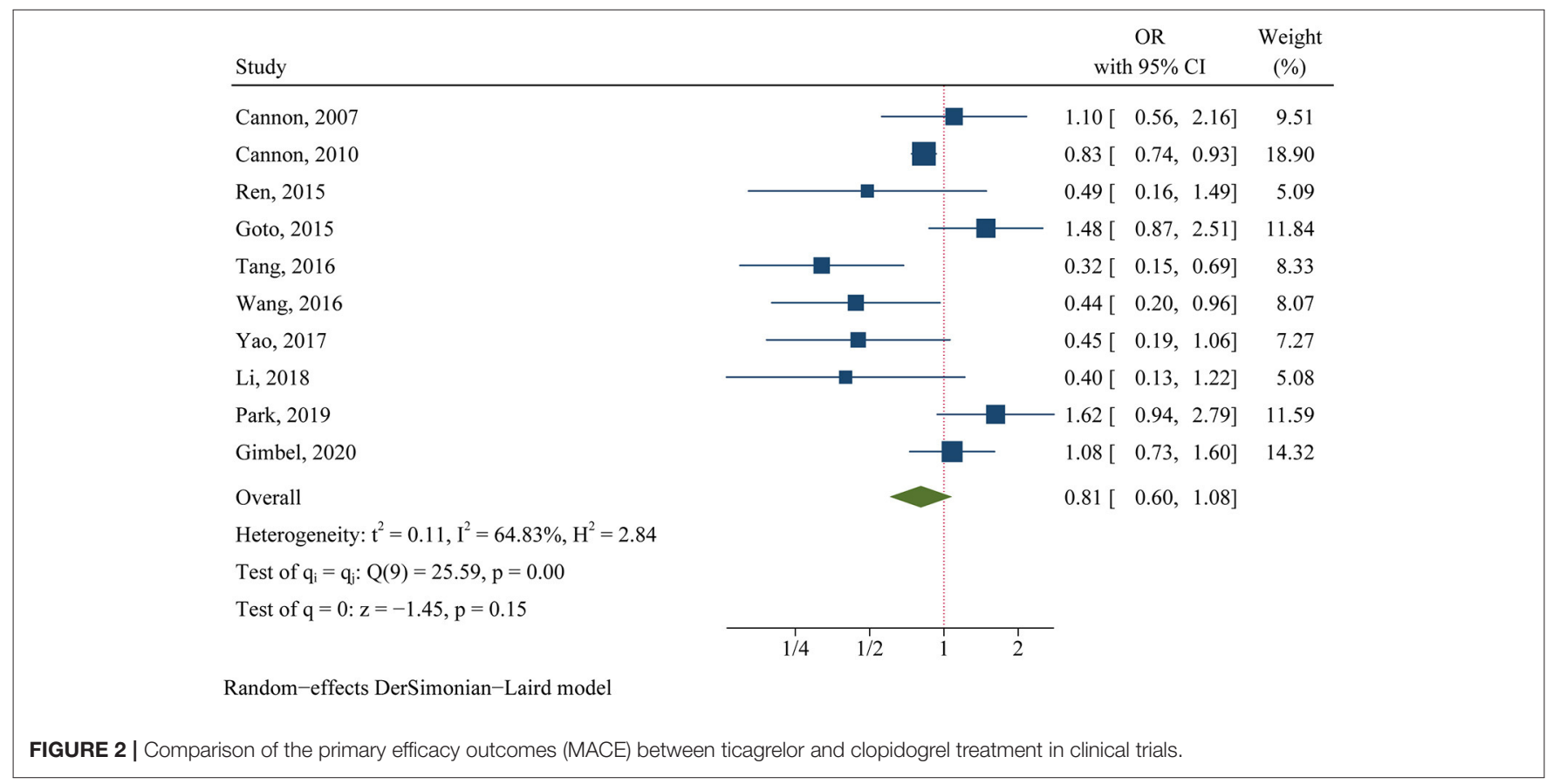




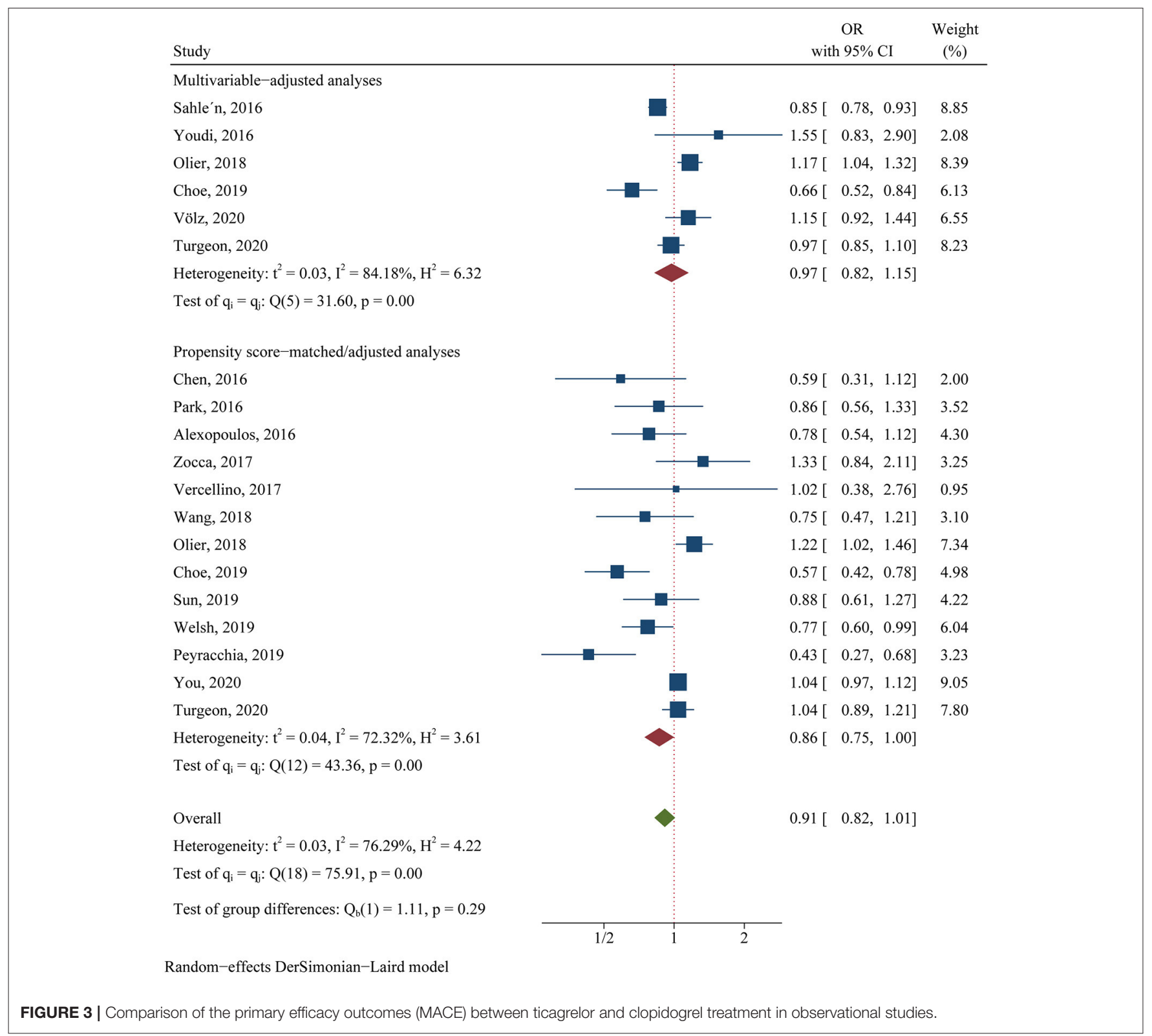

and Japan, and European and American countries, such as the United States, Canada, Sweden, the Netherlands, and England. In addition, the ethnicities of the cohorts were East Asians and Caucasians. And the duration of follow-up ranged from 30 to 468 days. The main studies and population characteristics are summarized in Supplementary Tables S3, S4.

\section{Efficacy Endpoints}

For the clinical trials, no significant difference was found in the primary efficacy endpoint (MACE) between ticagrelor and clopidogrel groups (OR 0.81, 95\% CI 0.60-1.08, $p=0.15, I^{2}=$ $64.83 \%$; Figure 2). For the observational studies, similar results were obtained in both the MA group (OR 0.97, 95\% CI 0.82-1.15, $p=0.76, I^{2}=84.18 \%$; Figure 3 ) and the PA group (OR 0.86, 95\% CI $0.75-1.00, p=0.05, I^{2}=72.32 \%$; Figure 3 ).
Compared with the clopidogrel group, the ticagrelor group demonstrated a reduction in secondary endpoints, including ST (OR 0.72, 95\% CI 0.58-0.90, $p=0.00, I^{2}=0.00 \%$; Table 1) in clinical trials, all-cause death (OR 0.83, 95\% CI 0.70-0.98 $p=$ $0.03, I^{2}=69.89 \%$ ) and CV death (OR 0.66, 95\% CI 0.44-0.99, $p=0.04, I^{2}=70.59 \%$ ) in the PA group, and CV death (OR 0.59, $95 \%$ CI $0.45-0.79, p<0.001$ ) in the MA group (Table 2).

\section{Safety Endpoints}

Ticagrelor led to significantly higher risks of bleeding (OR 1.46, 95\% CI 1.17-1.83, $p=0.00, I^{2}=61.66 \%$ ) and minor bleeding (OR $1.71,95 \%$ CI $\left.1.33-2.21, p=0.00, I^{2}=4.65 \%\right)$ over clopidogrel in clinical trials (Table 1). The increased risks of bleeding (OR 1.39, 95\% CI $\left.1.06-1.83, p=0.02, I^{2}=76.11 \%\right)$ and minor bleeding (OR 1.61, 95\% CI 1.37-1.89, $p=0.00, I^{2}=0.00 \%$ ) were also 
identified in the PA group of observational studies (Table 2). However, only the minor bleeding risk (OR 1.21, 95\% CI 1.14$1.72, p=0.007$; Table 2) increased significantly in the MA group of observational studies.

\section{Subgroup Analysis}

In the subgroup of PCI strategy, patients underwent PCI (OR $0.38,95 \%$ CI $0.23-0.63, p=0.00, I^{2}=0$ ) benefited more from ticagrelor than those intended for PCI (OR 1.03, 95\% CI 0.76$1.38, p=0.87, I^{2}=64.26 \%$ ) regarding MACE (Table 3). However, ticagrelor introduced a higher risk of bleeding in the patients either underwent PCI (OR 1.64, 95\% CI 1.07-2.51, $p=0.02, I^{2}$ $=0$ ) or intended for PCI (OR 1.44 95\% CI 1.11-1.86, $p=0.01$, $I^{2}=68.56 \%$; Table 3).

Subgroup analysis based on different ethnicities was performed to compare the clinical outcomes of ticagrelor and clopidogrel in Caucasian and East Asian populations, respectively. Ticagrelor showed a superior MACE reducing effect (OR 0.84, 95\% CI 0.75-0.94, $p=0.00, I^{2}=0$; Table 3) and a lower risk of bleeding (OR 1.09, 95\% CI 0.99-1.20, $p=$ $0.07, I^{2}=0$; Table 3 ) over clopidogrel in Caucasian patients. However, the results were inconsistent in East Asian populations.

TABLE 1 | Comparison of ticagrelor and clopidogrel treatment for the safety and second efficacy endpoints in clinical trials.

\begin{tabular}{lcccc}
\hline Outcomes & Trials & OR $(\mathbf{9 5 \%} \mathbf{C l})$ & $\mathbf{I}^{\mathbf{2}}$ & $\boldsymbol{p}$ Value \\
\hline Bleeding & 8 & $1.46(1.17,1.83)$ & 61.66 & 0.00 * \\
Major bleeding & 7 & $1.22(0.93,1.61)$ & 49.05 & 0.14 \\
Minor bleeding & 6 & $1.71(1.33,2.21)$ & 4.65 & $0.00^{*}$ \\
All-cause death & 8 & $0.85(0.71,1.01)$ & 4.05 & 0.07 \\
CV death & 8 & $0.87(0.63,1.22)$ & 30.86 & 0.43 \\
Ml & 8 & $0.87(0.66,1.16)$ & 39.12 & 0.36 \\
Stroke & 8 & $1.08(0.78,1.49)$ & 4.41 & 0.64 \\
Stent thrombosis & 3 & $0.72(0.58,0.90)$ & 0.00 & $0.00^{*}$ \\
\hline
\end{tabular}

"significant p-value compared with clopidogrel group. CV death, cardiovascular death; MI, myocardial infarction.
Ticagrelor was comparable with clopidogrel regarding MACE (OR 0.67, 95\% CI 0.36-1.25, $p=0.21, I^{2}=77.78 \%$; Table 3) and introduced a higher bleeding risk (OR 1.81, 95\% CI 1.43-2.29, $p$ $=0.00, I^{2}=0$; Table 3 ).

Subgroup analysis based on different Asian countries showed that Chinese patients benefited more from ticagrelor than those in Korean and Japanese, while the bleeding risk of ticagrelor significantly increased in all three Asian countries (Table 3). Further subgroup analysis was conducted to analyze the safety and efficacy of ticagrelor and clopidogrel based on different follow-up duration. It showed MACE was comparable between the two groups during the follow-up duration, while the bleeding risk of both two groups increased with a longer follow-up duration (Table 3).

Ticagrelor became widely available in 2012 (43). We made a subgroup analysis about the efficacy and safety of ticagrelor to detect the time effect. There was a lower MACE incidence before 2012, mainly relying on the lower incidences of CV death, MI, and ST under ticagrelor treatment while the bleeding risk was acceptable. After 2012, both the incidence of MACE and the second efficacy endpoints were comparable between the two groups, but the risk of bleeding and minor bleeding was higher than clopidogrel (Supplementary Table S8).

\section{Sensitivity Analyses and Publication Bias}

Sensitivity analyses were performed by including high-quality RCTs and by only including studies with clinical event committee-adjudicated events in RCTs. The results remained consistent (Supplementary Tables S9, S10).

According to different data types, publication bias was investigated in the two pairings: the PA group and clinical trials, and the MA group and clinical trials. By funnel plots (Figure 4) and Egger's test (Table 4), we detected publication only in MACE and MI between the PA group and clinical trials. The results of the non-parametric trim-and-fill analysis showed that five studies were filled for MACE with the total results influenced, and two were filled for MI with the total results unaffected (Supplementary Table S11).

TABLE 2 | Comparison of ticagrelor and clopidogrel treatment for the safety and second efficacy endpoints in observational studies.

\begin{tabular}{|c|c|c|c|c|c|c|c|c|}
\hline \multirow[t]{2}{*}{ Outcomes } & \multicolumn{4}{|c|}{ PA group } & \multicolumn{4}{|c|}{ MA group } \\
\hline & Studies & OR (95\%Cl) & $I^{2}$ & $p$ Value & Studies & OR (95\%Cl) & $I^{2}$ & $p$ Value \\
\hline Bleeding & 7 & $1.39(1.06,1.83)$ & 76.11 & $0.02^{*}$ & 6 & $1.15(0.86,1.53)$ & 81.88 & 0.35 \\
\hline Major bleeding & 8 & $1.26(0.90,1.75)$ & 75.23 & 0.17 & 2 & $1.12(0.86,1.45)$ & 0.00 & 0.39 \\
\hline Minor bleeding & 4 & $1.61(1.37,1.89)$ & 0.00 & 0.00 * & 1 & $1.21(1.14,1.72)$ & - & $0.007^{\star}$ \\
\hline All-cause death & 13 & $0.83(0.70,0.98)$ & 69.89 & $0.03^{*}$ & 8 & $0.93(0.78,1.10)$ & 79.69 & 0.38 \\
\hline CV death & 6 & $0.66(0.44,0.99)$ & 70.59 & $0.04^{*}$ & 1 & $0.59(0.45,0.79)$ & - & $<0.001^{\star}$ \\
\hline $\mathrm{Ml}$ & 11 & $0.99(0.84,1.15)$ & 48.41 & 0.87 & 4 & $0.90(0.71,1.15)$ & 79.13 & 0.39 \\
\hline Stroke & 11 & $0.84(0.65,1.09)$ & 39.97 & 0.19 & 4 & $0.79(0.59,1.06)$ & 52.70 & 0.12 \\
\hline Stent thrombosis & 5 & $1.18(0.81,1.72)$ & 6.26 & 0.39 & 2 & $1.45(0.89,2.37)$ & 0,00 & 0.14 \\
\hline
\end{tabular}

"significant p value compared with clopidogrel group. CV death, cardiovascular death; MI, myocardial infarction; PA group, propensity score-matched/adjusted analyses; MA group, multivariable-adjusted analyses. 
TABLE 3 | Subgroup comparison for MACE and bleeding outcomes between ticagrelor and clopidogrel treatment in RCTs.

\begin{tabular}{|c|c|c|c|c|c|c|c|c|}
\hline \multirow[t]{2}{*}{ Outcomes } & \multicolumn{2}{|c|}{ Subgroup } & \multirow[t]{2}{*}{ Trials } & \multicolumn{2}{|c|}{ Participants } & \multirow[t]{2}{*}{ OR (95\% Cl) } & \multirow[t]{2}{*}{$\mathbf{I}^{2}$} & \multirow[t]{2}{*}{$p$ Value } \\
\hline & & & & Ticagrelor & Clopidogrel & & & \\
\hline \multirow[t]{13}{*}{ MACE } & $\mathrm{PCl}$ strategy & & & & & & & \\
\hline & & Intended for $\mathrm{PCl}$ & 6 & 8,469 & 8,403 & $1.03(0.76,1.38)$ & 64.26 & 0.87 \\
\hline & & Underwent PCl & 3 & 421 & 541 & $0.38(0.23,0.63)$ & 0.00 & $0.00^{\star}$ \\
\hline & Ethnicity & & & & & & & \\
\hline & & East Asian & 6 & 1,322 & 1,441 & $0.67(0.36,1.25)$ & 77.78 & 0.21 \\
\hline & & Caucasian & 2 & 7,066 & 7,003 & $0.84(0.75,0.94)$ & 0.00 & $0.00^{\star}$ \\
\hline & Asian countries & China & 4 & 521 & 641 & $0.40(0.26,0.60)$ & 0.00 & 0.00 * \\
\hline & & Korea & 1 & 400 & 400 & $1.62(0.94,2.79)$ & - & 0.07 \\
\hline & & Japan\# & 1 & 401 & 400 & $1.48(0.87,2.51)$ & - & 0.15 \\
\hline & Follow-up duration & $<=1$ & 1 & 334 & 327 & $1.05(0.52,2.52)$ & - & 0.71 \\
\hline & & $>1$ & 9 & 8,890 & 8,944 & $0.83(0.61,1.12)$ & 67.54 & 0.22 \\
\hline & & $<=6$ & 4 & 928 & 914 & $0.66(0.35,1.26)$ & 63.85 & 0.21 \\
\hline & & $>6$ & 6 & 8,296 & 8,357 & $0.96(0.69,1.33)$ & 67.66 & 0.79 \\
\hline \multirow[t]{14}{*}{ Bleeding } & PCl strategy & & & & & & & \\
\hline & & Intended for $\mathrm{PCl}$ & 6 & 8,469 & 8,403 & $1.44(1.11,1.86)$ & 68.56 & $0.01^{*}$ \\
\hline & & Underwent PCl & 2 & 361 & 481 & $1.64(1.07,2.51)$ & 0.00 & $0.02^{*}$ \\
\hline & Ethnicity & & & & & & & \\
\hline & & East Asian & 5 & 1,262 & 1,381 & $1.81(1.43,2.29)$ & 0.00 & $0.00^{*}$ \\
\hline & & Caucasian & 2 & 7,066 & 7,003 & $1.09(0.99,1.20)$ & 0.00 & 0.07 \\
\hline & Asian countries & China & 3 & 461 & 581 & $1.64(1.13,2.37)$ & 0.00 & $0.01^{*}$ \\
\hline & & Korea & 1 & 400 & 400 & $2.29(1.34,3.92)$ & - & $0.02^{*}$ \\
\hline & & Japan ${ }^{\#}$ & 1 & 401 & 400 & $1.80(0.87,2.51)$ & - & $0.001^{\star}$ \\
\hline & Follow-up duration & & & & & & & \\
\hline & & $<=1$ & 1 & 334 & 327 & $1.23(0.71,2.12)$ & 0.00 & 0.62 \\
\hline & & $>1$ & 8 & 8,830 & 8,884 & $1.46(1.17,1.83)$ & 61.66 & $0.00^{*}$ \\
\hline & & $<=6$ & 3 & 868 & 854 & $1.23(0.88,1.72)$ & 0.00 & 0.22 \\
\hline & & $>6$ & 6 & 8,296 & 8,357 & $1.54(1.17,2.02)$ & 71.98 & $0.00^{*}$ \\
\hline
\end{tabular}

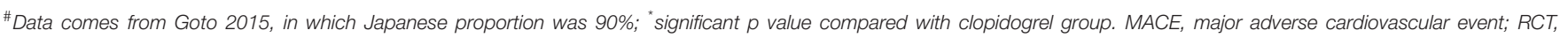
randomized controlled trials; $\mathrm{PCl}$, percutaneous coronary intervention.

\section{DISCUSSION}

This meta-analysis, based on 28 studies, suggested that ticagrelor was not only inferior to clopidogrel in patients with ACS but also related to an increased bleeding risk, whereas ticagrelor was more effective for the patients who underwent PCI than clopidogrel, as it significantly reduced the incidence of MACE. Meanwhile, this meta-analysis revealed that Caucasians and East Asians had inconsistent safety and efficacy profiles under ticagrelor treatment. Among East Asian patients, Chinese benefited more from ticagrelor than Korean and Japanese. Care should, therefore, be taken to screen the eligible population when applying ticagrelor and the issue of increased bleeding risk with longer follow-up duration under ticagrelor treatment was of concern.

Recently, several studies have revisited the issues concerning clinical applications of ticagrelor and clopidogrel. These metaanalyses got no consensus on the efficacy and safety of ticagrelor treatment. They either mixed all studies together and ignored the heterogeneity between clinical trials and observational studies, or did not perform further subgroup analyses based on other studies (or population) characteristics $(13,15,16)$. In this metaanalysis, we performed prespecified subgroup analyses for MACE and bleeding in RCTs according to PCI strategy, ethnicity, country, and follow-up duration. Additionally, we performed two subgroup analyses (the PA and MA groups) in observational studies according to different data types. We conducted a comprehensive analysis from both clinical trial and real-world practice considerations to compare the clinical outcomes of ticagrelor and clopidogrel in different subgroups. Therefore, some definitive evidence can be provided for clinicians to choose between ticagrelor and clopidogrel.

We found similar primary efficacy results of ticagrelor treatment in both clinical trials and observational studies, though the result of the MA group differed slightly. The results of the secondary efficacy endpoints differed in clinical trials and observational studies, which can be attributed to inherent differences between study types. In brief, clinical trials (especially RCTs) match baseline characteristics well. However, although clinical factors associated with treatment selection 
A
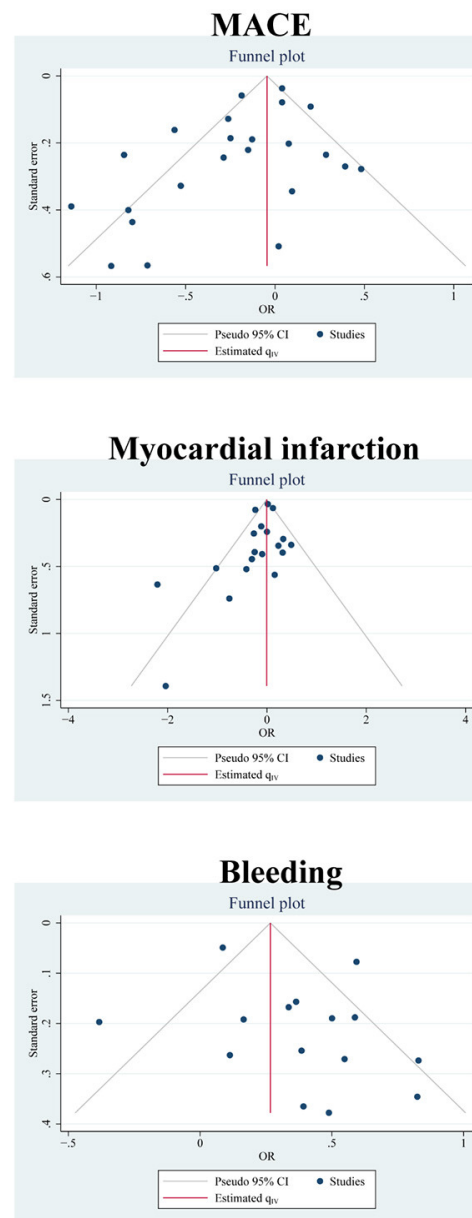

B
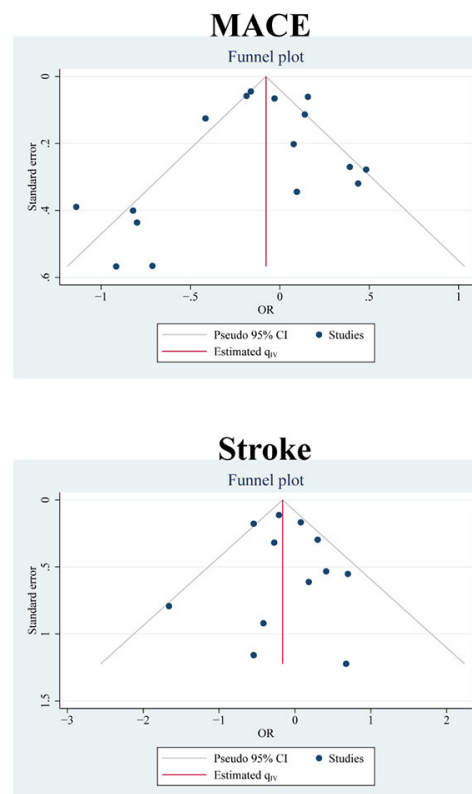
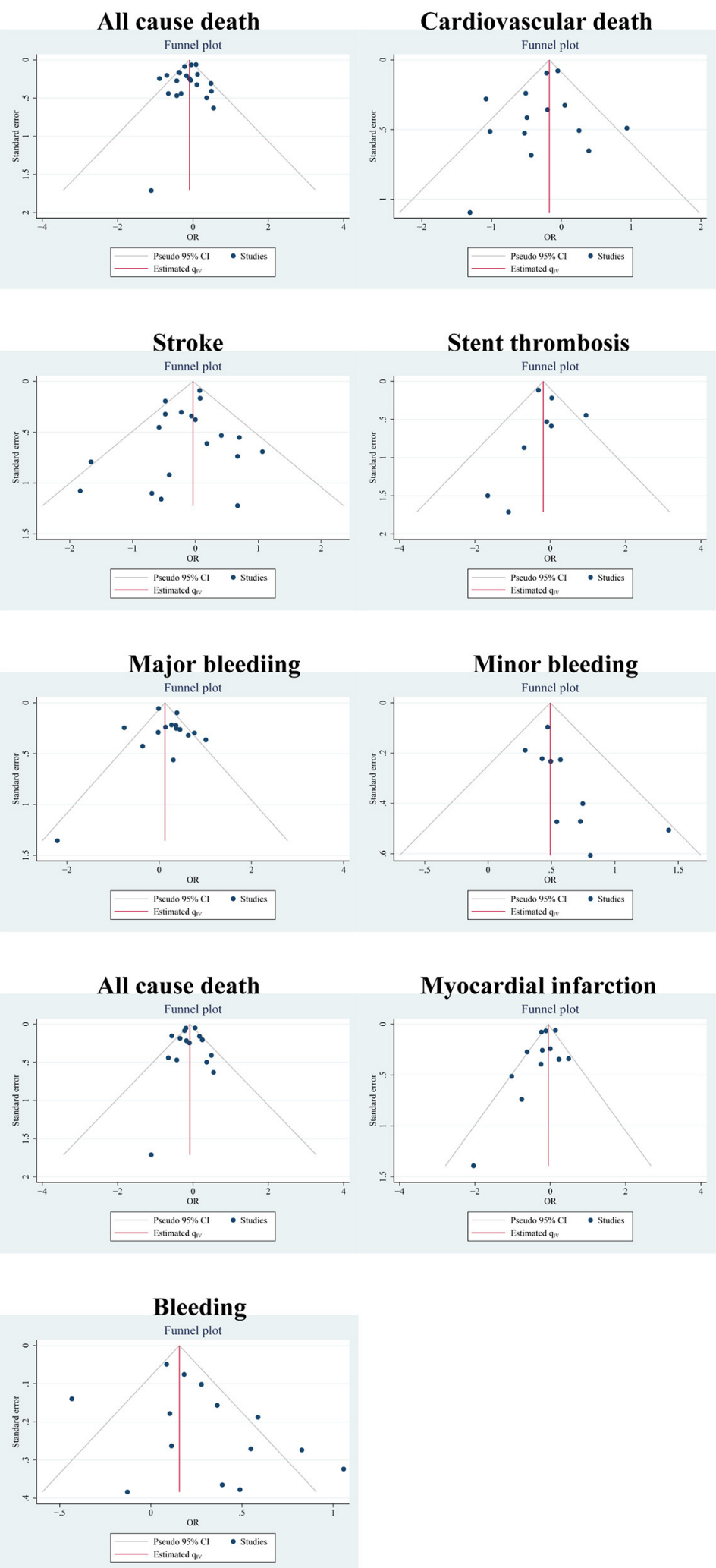

FIGURE 4 | Publication bias of outcomes using funnel plots: (A) in the PA group and clinical trials; (B) in the MA group and clinical trials. 
TABLE 4 | Publication bias of outcomes using the Egger's test.

\begin{tabular}{|c|c|c|c|c|}
\hline \multirow[t]{2}{*}{ Outcomes } & \multicolumn{2}{|c|}{ PA group and clinical trials } & \multicolumn{2}{|c|}{ MA group and clinical trials } \\
\hline & Studies & Prob > |z| & Studies & Prob $>|z|$ \\
\hline MACE & 23 & $0.0197^{\star}$ & 16 & 0.1163 \\
\hline All-cause death & 21 & 0.9956 & 16 & 0.7295 \\
\hline CV death & 14 & 0.7616 & 9 & NA \\
\hline $\mathrm{Ml}$ & 20 & $0.0486^{*}$ & 13 & 0.1195 \\
\hline Stoke & 19 & 0.7060 & 12 & 0.7660 \\
\hline Stent thrombosis & 10 & 0.5747 & 7 & NA \\
\hline Bleeding & 15 & 0.3814 & 14 & 0.0718 \\
\hline Major bleeding & 15 & 0.4940 & 9 & NA \\
\hline Minor bleeding & 10 & 0.1490 & 7 & NA \\
\hline
\end{tabular}

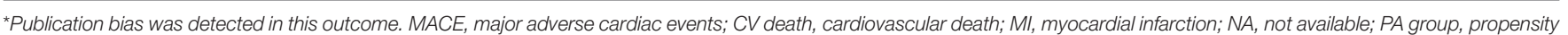
score-matched/adjusted analyses; MA group, multivariable-adjusted analyses.

in observational studies can be matched by propensity scorematched/adjusted analyses or multivariable-adjusted analyses, there are still some unadjusted or incomplete adjustment variables that may affect the results. This may explain the inconsistent results of bleeding in the MA group and the secondary endpoints in clinical studies and observational studies.

In addition to pharmacological treatment with DAPT, the primary management of patients with ACS involves early invasive strategies, namely, coronary angiography, PCI, and even coronary artery bypass grafting (CABG) $(2,4,44)$. Considering that many high-risk patients with ACS received PCI after coronary angiography, we included those who underwent or intended for PCI to better compare the clinical outcomes. Subgroup comparison in this study revealed that ticagrelor could significantly reduce the incidence of MACE in patients who underwent PCI compared with those intended for PCI. Patients with ACS who received PCI or CABG had different clinical characteristics from those who received medical treatment alone. Platelet activation plays a significant role in PCIrelated thrombotic events. Intra-individual variability in platelet reactivity occurred in patients undergoing elective coronary stenting (45). In addition, high clopidogrel on-treatment platelet reactivity (HTPR) enhanced the occurrence of ischemic adverse events (46). On the contrary, ticagrelor produces a more potent antiplatelet effect than clopidogrel. Bonello et al. reported that ticagrelor induced an optimal platelet reactivity inhibition in patients who underwent PCI (47), and it provided an approach for overcoming HTPR (48). Patients who underwent PCI benefit more from ticagrelor treatment than those intended for PCI, providing valuable suggestions for clinicians to choose between ticagrelor and clopidogrel according to the PCI strategy.

In terms of ethnicity, Caucasians made up $91 \%$ of the PLATO trial population, whereas East Asians accounted for only 6\%. Caucasians and East Asians differ substantially in phenotypes and genomics. Therefore, recommendations based on Caucasians do not necessarily apply to East Asians (13). We conducted subgroup analyses in Caucasians and East Asians, respectively. The results were different between the two subgroups. East Asians are thought to be more prone to bleeding events than Caucasians but are relatively resistant to adverse ischemic outcomes after PCI (the so-called "East Asian paradox") (49-51). In addition, cytochrome P450 2C19 lossof-function alleles associated with high platelet reactivity are more common in Asian populations (52). In a nutshell, there are significant ethnic differences between Caucasian and East Asian patients in terms of thrombosis, platelet P2Y12 receptor inhibition, and predisposition to bleeding complications (49). Those differences might partly contribute to this interpopulation disparity. Considering the difference between ischemia and bleeding risk, the choice of antithrombotic drugs may differ between East Asian patients and Caucasian patients. The unique risk-benefit trade-off in East Asian patients was worthy of attention. The number of RCTs included in this metaanalysis is relatively small, and more RCTs are required for further elaboration.

In East Asia, the MACE incidence was significantly reduced by ticagrelor in Chinese compared with Korean and Japanese, which may be partially attributed to the different proportions of patients who underwent PCI. Specifically, $81.5 \%$ of the patients with ACS in the Korean TICAKOREA trial (8) and $84.8 \%$ of the patients with ACS in the Japanese PHILO trial (9) were assigned to the ticagrelor group underwent PCI. More importantly, data from four Chinese trials showed that up to $94 \%$ of the patients with ACS in the ticagrelor group underwent PCI. Additionally, the baseline characteristics, diagnostic criteria, and dosage regimen might be the reasons for the differences. The efficacy of ticagrelor in Asian populations needs to be validated by larger trials.

In this meta-analysis, the primary endpoint of MACE was comparable between ticagrelor and clopidogrel in the patients with ACS. However, ticagrelor introduced an increased bleeding risk with the extension of follow-up duration, which deserves attention. Researchers have explored the possibility of a shorterduration DAPT. Abbreviated-duration ( $\leq 6$ months) DAPT in the CAD population did not significantly increase the incidence of MACE, but dramatically reduced the risk of major bleeding (53). Additionally, the safety and efficacy of short-duration ( $\leq$ 3 months) DAPT in elderly patients were also acceptable (54). However, the optimal DAPT duration after PCI still remains a topic of emerging interest. There are growing concerns regarding P2Y12 inhibitor monotherapy. Valgimigli et al. proved that, 
compared with DAPT, P2Y12 inhibitor monotherapy was noninferior in efficacy endpoints, and with a reduced risk of major bleeding (55). Considering the reduced bleeding risk, P2Y12 inhibitor monotherapy should be considered after a shortduration DAPT, based on the individual's ischemia and bleeding risks (56). Thus, balancing the risks of ischemia and bleeding when using DAPT has always been a clinical challenge.

This study has several limitations. First, the sample sizes of several RCTs and the number of RCTs are relatively small. Second, the follow-up duration subsequent to PCI varied from in-hospital to one year or longer. Although we performed subgroup analysis in terms of the duration, the total results might also have swayed to some extent. Third, we pooled trials with heterogeneous populations that varied in study design, disease subtype, treatment strategy, and endpoint definition. Fourth, possible adverse drug reactions, loss of tolerability, and discontinuation of treatment are not incorporated into the consideration.

\section{CONCLUSION}

We suggest that ticagrelor has a comparable efficacy but a higher risk of bleeding compared to clopidogrel for patients with ACS. Clinicians should selectively adopt ticagrelor or clopidogrel according to different PCI strategies, ethnicities, and countries.

\section{REFERENCES}

1. Joseph P, Leong D, McKee M, Anand SS, Schwalm JD, Teo K, et al. Reducing the Global Burden of Cardiovascular Disease, Part 1: The Epidemiology and Risk Factors. Circ Res. (2017) 121:677-94. doi: 10.1161/CIRCRESAHA.117.308903

2. Sousa-Uva M, Neumann FJ, Ahlsson A, Alfonso F, Banning AP, Benedetto U, et al. 2018 ESC/EACTS Guidelines on myocardial revascularization. Eur J Cardiothorac Surg. (2019) 55:4-90. doi: 10.15829/1560-4071-2019-8-151-226

3. Kamran H, Jneid H, Kayani WT, Virani SS, Levine GN, Nambi V, et al. Oral antiplatelet therapy after acute coronary syndrome: a review. Jama. (2021) 325:1545-55. doi: 10.1001/jama.2021.0716

4. Levine GN, Bates ER, Bittl JA, Brindis RG, Fihn SD, Fleisher LA, et al. 2016 ACC/AHA Guideline Focused Update on Duration of Dual Antiplatelet Therapy in Patients With Coronary Artery Disease: A Report of the American College of Cardiology/American Heart Association Task Force on Clinical Practice Guidelines: An Update of the 2011 ACCF/AHA/SCAI Guideline for Percutaneous Coronary Intervention, 2011 ACCF/AHA Guideline for Coronary Artery Bypass Graft Surgery, 2012 ACC/AHA/ACP/AATS/PCNA/SCAI/STS Guideline for the Diagnosis and Management of Patients With Stable Ischemic Heart Disease, 2013 ACCF/AHA Guideline for the Management of ST-Elevation Myocardial Infarction, 2014 AHA/ACC Guideline for the Management of Patients With Non-ST-Elevation Acute Coronary Syndromes, and 2014 ACC/AHA Guideline on Perioperative Cardiovascular Evaluation and Management of Patients Undergoing Noncardiac Surgery. Circulation. (2016) 134:e123-55. doi: 10.1161/CIR.00000000000 00404

5. Cannon CP, Husted S, Harrington RA, Scirica BM, Emanuelsson H, Peters G, et al. Safety, tolerability, and initial efficacy of AZD6140, the first reversible oral adenosine diphosphate receptor antagonist, compared with clopidogrel, in patients with non-ST-segment elevation acute coronary syndrome: primary results of the DISPERSE-2 trial. J Am Coll Cardiol. (2007) 50:184451. doi: 10.1016/j.jacc.2007.07.053

\section{DATA AVAILABILITY STATEMENT}

The raw data supporting the conclusions of this article will be made available by the authors, without undue reservation.

\section{AUTHOR CONTRIBUTIONS}

LL contributed to the conception or design of the study. MS, WC, and LL contributed to the acquisition, analysis, or interpretation of data for the study. MS and WC drafted the manuscript. LL critically revised the manuscript. All authors gave final approval and agree to be accountable for all aspects of work ensuring integrity and accuracy.

\section{ACKNOWLEDGMENTS}

We thank TopEdit (www.topeditsci.com) for its linguistic assistance during the preparation of this manuscript.

\section{SUPPLEMENTARY MATERIAL}

The Supplementary Material for this article can be found online at: https://www.frontiersin.org/articles/10.3389/fcvm. 2021.818215/full\#supplementary-material

6. Tantry US, Navarese EP, Myat A, Gurbel PA. Selection of P2Y12 Inhibitor in Percutaneous Coronary Intervention and/or Acute Coronary Syndrome. Prog Cardiovasc Dis. (2018) 60:460-70. doi: 10.1016/j.pcad.2018.01.003

7. Cannon CP, Harrington RA, James S, Ardissino D, Becker RC, Emanuelsson $\mathrm{H}$, et al. Comparison of ticagrelor with clopidogrel in patients with a planned invasive strategy for acute coronary syndromes (PLATO): a randomised double-blind study. Lancet. (2010) 375:283-93. doi: 10.1016/S0140-6736(09)62191-7

8. Park DW, Kwon O, Jang JS, Yun SC, Park H, Kang DY, et al. Clinically significant bleeding with ticagrelor versus clopidogrel in korean patients with acute coronary syndromes intended for invasive management: a randomized clinical trial. Circulation. (2019) 140:1865-77. doi: 10.1161/CIRCULATIONAHA.119.041766

9. Goto S, Huang CH, Park SJ, Emanuelsson H, Kimura T. Ticagrelor vs. clopidogrel in Japanese, Korean and Taiwanese patients with acute coronary syndrome - randomized, double-blind, phase III PHILO study. Circ J. (2015) 79:2452-60. doi: 10.1253/circj.CJ-15-0112

10. Sahlén A, Varenhorst C, Lagerqvist B, Renlund H, Omerovic E, Erlinge D, et al. Outcomes in patients treated with ticagrelor or clopidogrel after acute myocardial infarction: Experiences from SWEDEHEART registry. Eur Heart J. (2016) 37:3335-42. doi: 10.1093/eurheartj/ehw284

11. Olier I, Sirker A, Hildick-Smith DJR, Kinnaird T, Ludman P, De Belder $\mathrm{MA}$, et al. Association of different antiplatelet therapies with mortality after primary percutaneous coronary intervention. Heart. (2018) 104:168390. doi: 10.1136/heartjnl-2017-312366

12. You SC, Rho Y, Bikdeli B, Kim J, Siapos A, Weaver J, et al. Association of ticagrelor vs. clopidogrel with net adverse clinical events in patients with acute coronary syndrome undergoing percutaneous coronary intervention. JAMA. (2020) 324:1640-50. doi: 10.1001/jama.2020. 16167

13. Wu H, Xiang X, Li D, Shen S, Li X. Efficacy and safety of ticagrelor compared to clopidogrel in patients undergoing percutaneous coronary intervention: a meta-analysis. Curr Pharm Des. (2020) 26:5988-97. doi: 10.2174/1381612826666200614184007 
14. Watti H, Dahal K, Zabher HG, Katikaneni P, Modi K, Abdulbaki A. Comparison of prasugrel and ticagrelor in patients with acute coronary syndrome undergoing percutaneous coronary intervention: a meta-analysis of randomized and non-randomized studies. Int J Cardiol. (2017) 249:6672. doi: 10.1016/j.ijcard.2017.07.103

15. Guan W, Lu H, Yang K. Choosing between ticagrelor and clopidogrel following percutaneous coronary intervention: a systematic review and meta-analysis (2007-2017). Medicine. (2018) 97:e12978. doi: 10.1097/MD.0000000000012978

16. Fan ZG, Zhang WL, Xu B, Ji J, Tian NL, He SH. Comparisons between ticagrelor and clopidogrel following percutaneous coronary intervention in patients with acute coronary syndrome: a comprehensive meta-analysis. Drug Des Devel Ther. (2019) 13:719-30. doi: 10.2147/DDDT.S196535

17. Moher D, Liberati A, Tetzlaff J, Altman DG. Preferred reporting items for systematic reviews and meta-analyses: the PRISMA statement. Int J Surg. (2010) 8:336-41. doi: 10.1016/j.ijsu.2010.02.007

18. Sterne JAC, Savović J, Page MJ, Elbers RG, Blencowe NS, Boutron I, et al. RoB 2: a revised tool for assessing risk of bias in randomised trials. BMJ. (2019) 366:14898. doi: 10.1136/bmj.14898

19. Stang A. Critical evaluation of the Newcastle-Ottawa scale for the assessment of the quality of nonrandomized studies in meta-analyses. Eur J Epidemiol. (2010) 25:603-5. doi: 10.1007/s10654-010-9491-Z

20. Chaimani A, Higgins JP, Mavridis D, Spyridonos P, Salanti G. Graphical tools for network meta-analysis in STATA. PLoS One. (2013) 8:e76654. doi: 10.1371/journal.pone.0076654

21. Leonardi S, Branca M, Franzone A, McFadden E, Piccolo R, Jüni P, et al. Comparison of investigator-reported and clinical event committeeadjudicated outcome events in GLASSY. Circ Cardiovasc Qual Outcomes. (2021) 14:e006581. doi: 10.1161/CIRCOUTCOMES.120.006581

22. Ren Q, Ren C, Liu X, Dong C, Zhang X. Ticagrelor vs. clopidogrel in non-ST-elevation acute coronary syndromes. Herz. (2016) 41:2469. doi: 10.1007/s00059-015-4359-3

23. Tang $\mathrm{X}$, Li R, Jing Q, Wang Q, Liu $\mathrm{P}$, Zhang $\mathrm{P}$, et al. Assessment of ticagrelor versus clopidogrel treatment in patients with STelevation myocardial infarction undergoing primary percutaneous coronary intervention. J Cardiovasc Pharmacol. (2016) 68:11520. doi: 10.1097/FJC.0000000000000390

24. Wang H, Wang X. Efficacy and safety outcomes of ticagrelor compared with clopidogrel in elderly Chinese patients with acute coronary syndrome. Ther Clin Risk Manag. (2016) 12:1101-5. doi: 10.2147/TCRM.S108965

25. Yao Z, Li G, Fu C, Li G. Analysis of antiplatelet activity and short-term prognosis of ticagrelor in AMI patients undergoing emergency PCI during perioperative period. Int J Clin Exper Med. (2017) 10:9595-600.

26. Li XY, Su GH, Wang GX, Hu HY, Fan CJ. Switching from ticagrelor to clopidogrel in patients with ST-segment elevation myocardial infarction undergoing successful percutaneous coronary intervention in real-world China: occurrences, reasons, and long-term clinical outcomes. Clin Cardiol. (2018) 41:1446-54. doi: 10.1002/clc.23074

27. Gimbel M, Qaderdan K, Willemsen L, Hermanides R, Bergmeijer T, de Vrey E, et al. Clopidogrel versus ticagrelor or prasugrel in patients aged 70 years or older with non-ST-elevation acute coronary syndrome (POPular AGE): the randomised, open-label, non-inferiority trial. Lancet. (2020) 395:137481. doi: 10.1016/S0140-6736(20)30325-1

28. Alexopoulos D, Xanthopoulou I, Deftereos S, Hamilos M, Sitafidis G, Kanakakis I, et al. Contemporary antiplatelet treatment in acute coronary syndrome patients undergoing percutaneous coronary intervention: 1-year outcomes from the GReek AntiPlatElet (GRAPE) Registry. J Thrombosis Haemostasis. (2016) 14:1146-54. doi: 10.1111/jth.13316

29. Chen IC, Lee CH, Fang CC, Chao TH, Cheng CL, Chen Y, et al. Efficacy and safety of ticagrelor versus clopidogrel in acute coronary syndrome in Taiwan: a multicenter retrospective pilot study. J Chin Med Assoc. (2016) 79:521-30. doi: 10.1016/j.jcma.2016.02.010

30. Park KH, Jeong MH, Ahn Y, Ahn TH, Seung KB, Oh DJ, et al. Comparison of short-term clinical outcomes between ticagrelor versus clopidogrel in patients with acute myocardial infarction undergoing successful revascularization; from Korea Acute Myocardial Infarction Registry-National Institute of Health. Int J Cardiol. (2016) 215:193-200. doi: 10.1016/j.ijcard.2016.04.044
31. Yudi MB, Clark DJ, Farouque O, Eccleston D, Andrianopoulos N, Duffy SJ, et al. Clopidogrel, prasugrel or ticagrelor in patients with acute coronary syndromes undergoing percutaneous coronary intervention. Intern Med J. (2016) 46:559-65. doi: 10.1111/imj.13041

32. Vercellino M, Sànchez FA, Boasi V, Perri D, Tacchi C, Secco GG, et al. Ticagrelor versus clopidogrel in real-world patients with ST elevation myocardial infarction: 1-year results by propensity score analysis. BMC Cardiovasc Disor. (2017) 17:1-11. doi: 10.1186/s12872-017-0524-3

33. Zocca P, Van Der Heijden LC, Kok MM, Löwik MM, Hartmann M, Stoel MG, et al. Clopidogrel or ticagrelor in acute coronary syndrome patients treated with newer-generation drug-eluting stents: CHANGE DAPT. EuroIntervention. (2017) 13:1168-76. doi: 10.4244/EIJ-D-17-00634

34. Wang HY Li Y, Xu XM Li J, Han YL. Impact of baseline bleeding risk on efficacy and safety of ticagrelor versus clopidogrel in Chinese patients with acute coronary syndrome undergoing percutaneous coronary intervention. Chin Med J. (2018) 131:2017-24. doi: 10.4103/0366-6999.2 39306

35. Choe JC, Cha KS, Ahn J, Park JS, Lee HW, Oh JH, et al. Comparison of prescription rates and clinical outcomes in acute coronary syndrome patients who underwent percutaneous coronary intervention using different P2Y12 inhibitors in a large observational study. Int J Cardiol. (2019) 274:216. doi: 10.1016/j.ijcard.2018.09.011

36. Kim C, Shin DH, Hong SJ, Ahn CM, Kim JS, Kim BK, et al. One-year clinical outcomes of ticagrelor compared with clopidogrel after percutaneous coronary intervention in patients with acute myocardial infarction: from Korean Health Insurance Review and Assessment Data. J Cardiol. (2019) 73:191-7. doi: 10.1016/j.jjcc.2018.08.005

37. Krishnamurthy A, Keeble C, Anderson M, Somers K, BurtonWood N, Harland C, et al. Real-world comparison of clopidogrel, prasugrel and ticagrelor in patients undergoing primary percutaneous coronary intervention. Open Heart. (2019) 6:e000951. doi: 10.1136/openhrt-2018-000951

38. Sun Y, Li C, Zhang L, Yu T, Ye H, Yu B, et al. Clinical outcomes after ticagrelor and clopidogrel in Chinese post-stented patients. Atherosclerosis. (2019) 290:52-8. doi: 10.1016/j.atherosclerosis.2019. 09.011

39. Welsh RC, Sidhu RS, Cairns JA, Lavi S, Kedev S, Moreno R, et al. Outcomes Among Clopidogrel, Prasugrel, and Ticagrelor in ST-Elevation Myocardial Infarction Patients Who Underwent Primary Percutaneous Coronary Intervention From the TOTAL Trial. Canad J Cardiol. (2019) 35:1377-85. doi: 10.1016/j.cjca.2019.04.026

40. Peyracchia M, Saglietto A, Biolè C, Raposeiras-Roubin S, Abu-Assi E, Kinnaird T, et al. Efficacy and safety of clopidogrel, prasugrel and ticagrelor in ACS patients treated with PCI: a propensity score analysis of the RENAMI and BleeMACS registries. Am J Cardiovasc Drugs. (2020) 20:25969. doi: 10.1007/s40256-019-00373-1

41. Turgeon RD, Koshman SL, Youngson E, Har B, Wilton SB, James MT, et al. Association of ticagrelor vs clopidogrel with major adverse coronary events in patients with acute coronary syndrome undergoing percutaneous coronary intervention. JAMA Intern Med. (2020) 180:4208. doi: 10.1001/jamainternmed.2019.6447

42. Völz S, Petursson P, Odenstedt J, Ioanes D, Haraldsson I, Angerås $\mathrm{O}$, et al. Ticagrelor is not superior to clopidogrel in patients with acute coronary syndromes undergoing PCI: a report from Swedish coronary angiography and angioplasty registry. J Am Heart Assoc. (2020) 9:e015990. doi: 10.1161/JAHA.119.015990

43. Dayoub EJ, Seigerman M, Tuteja S, Kobayashi T, Kolansky DM, Giri J, et al. Trends in platelet adenosine diphosphate P2Y12 receptor inhibitor use and adherence among antiplatelet-naive patients after percutaneous coronary intervention, 2008-2016. JAMA Intern Med. (2018) 178:94350. doi: 10.1001/jamainternmed.2018.0783

44. Brilakis ES, Patel VG, Banerjee S. Medical management after coronary stent implantation: a review. Jama. (2013) 310:189-98. doi: 10.1001/jama.2013.7086

45. Gurbel PA, Bliden KP, Hiatt BL, O'Connor CM. Clopidogrel for coronary stenting: response variability, drug resistance, and the effect of pretreatment platelet reactivity. Circulation. (2003) 107:2908-13. doi: 10.1161/01.CIR.0000072771.11429.83 
46. Parodi G, Marcucci R, Valenti R, Gori AM, Migliorini A, Giusti B, et al. High residual platelet reactivity after clopidogrel loading and longterm cardiovascular events among patients with acute coronary syndromes undergoing PCI. Jama. (2011) 306:1215-23. doi: 10.1001/jama.2011.1332

47. Bonello L, Laine M, Thuny F, Paganelli F, Lemesle G, Roch A, et al. Platelet reactivity in patients receiving a maintenance dose of $\mathrm{P} 2 \mathrm{Y} 12-\mathrm{ADP}$ receptor antagonists undergoing elective percutaneous coronary intervention. Int $J$ Cardiol. (2016) 216:190-3. doi: 10.1016/j.ijcard.2016.04.165

48. Samoš M, Fedor M, Kovár F, Duraj L, Stančiaková L, Galajda P, et al. Ticagrelor: a safe and effective approach for overcoming clopidogrel resistance in patients with stent thrombosis? Blood Coagul Fibrinolysis. (2016) 27:11720. doi: 10.1097/MBC.0000000000000406

49. Levine GN, Jeong Y-H, Goto S, Anderson JL, Huo Y, Mega JL, et al. World heart federation expert consensus statement on antiplatelet therapy in east asian patients with ACS or undergoing PCI. Glob Heart. (2014) 9:45767. doi: 10.1016/j.gheart.2014.08.001

50. Kang J, Park KW, Palmerini T, Stone GW, Lee MS, Colombo A, et al. Racial differences in ischaemia/bleeding risk Trade-Off during anti-platelet therapy: individual patient level landmark meta-analysis from seven RCTs. Thromb Haemost. (2019) 119:149-62. doi: 10.1055/s-0038-1676545

51. Bae JS, Ahn J-H, Tantry US, Gurbel PA, Jeong Y-H. Should antithrombotic treatment strategies in East Asians differ from caucasians? Curr Vasc Pharmacol. (2018) 16:459-76. doi: 10.2174/1570161116666180117103238

52. Scott SA, Sangkuhl K, Stein CM, Hulot JS, Mega JL, Roden DM, et al. Clinical Pharmacogenetics Implementation Consortium guidelines for CYP2C19 genotype and clopidogrel therapy: 2013 update. Clin Pharmacol Ther. (2013) 94:317-23. doi: 10.1038/clpt.2013.105

53. Ziada KM, Abdel-Latif AK, Charnigo R, Moliterno DJ. Safety of an abbreviated duration of dual antiplatelet therapy ( $\leq 6$ months) following second-generation drug-eluting stents for coronary artery disease: a systematic review and meta-analysis of randomized trials. Catheter Cardiovasc Interv. (2016) 87:722-32. doi: 10.1002/ccd.26110
54. Roule V, Lemaitre A, Pommier W, Bignon M, Sabatier R, Blanchart K, et al. Safety and efficacy of very short dual antiplatelet therapy followed by P2Y12 inhibitor monotherapy in older patients undergoing percutaneous coronary intervention: meta-analysis of randomised controlled trials. Age Ageing. (2021) 50:1102-7. doi: 10.1093/ageing/afab047

55. Valgimigli M, Gragnano F, Branca M, Franzone A, Baber U, Jang $\mathrm{Y}$, et al. P2Y12 inhibitor monotherapy or dual antiplatelet therapy after coronary revascularisation: individual patient level meta-analysis of randomised controlled trials. BMJ. (2021) 373:n1332. doi: 10.1136/bmj. n1332

56. Collet JP, Thiele H, Barbato E, Barthélémy O, Bauersachs J, Bhatt DL, et al. 2020 ESC Guidelines for the management of acute coronary syndromes in patients presenting without persistent ST-segment elevation. Eur Heart J. (2021) 42:1289-367. doi: 10.1093/eurheartj/ehaa909

Conflict of Interest: The authors declare that the research was conducted in the absence of any commercial or financial relationships that could be construed as a potential conflict of interest.

Publisher's Note: All claims expressed in this article are solely those of the authors and do not necessarily represent those of their affiliated organizations, or those of the publisher, the editors and the reviewers. Any product that may be evaluated in this article, or claim that may be made by its manufacturer, is not guaranteed or endorsed by the publisher.

Copyright (c) 2022 Sun, Cui and Li. This is an open-access article distributed under the terms of the Creative Commons Attribution License (CC BY). The use, distribution or reproduction in other forums is permitted, provided the original author(s) and the copyright owner(s) are credited and that the original publication in this journal is cited, in accordance with accepted academic practice. No use, distribution or reproduction is permitted which does not comply with these terms. 